\title{
Packed red cell transfusion does not compromise chromosome analysis in newborns
}

Anita S. Kulharya, $P h D^{1}$, Bonnie A. Salbert, $D O^{3}$, Karen N. Norris ${ }^{1}$, Lloyd Cook, MD ${ }^{2}$, Patricia J. Larrison, $M T^{2}$, and David B. Flannery, $M D^{1}$

\begin{abstract}
Purpose: Critically ill neonates are frequently transfused with packed red cells. Some of these transfused neonates also need chromosome analysis. There is a long-standing tradition in pediatrics of not performing chromosome analysis after transfusion. We wished to determine whether transfusion with packed red cells affect the cytogenetic results in neonates. Method: The medical records of all neonates at the Medical College of Georgia who had had chromosome analysis between June 1995 and June 1998 were reviewed. Ten neonates had received transfusion prior to cytogenetic testing. Of these 10 infants, two had been transfused two or more times. Routine cytogenetic analysis of 20 metaphases at 550-band level had been performed on all 10 patients. Heteromorphic markers were compared in 10 randomly selected metaphases for any discrepancy. To determine whether there were theoretical reasons to delay chromosome analysis in transfused neonates, samples of irradiated, and/or filtered, and nonfiltered blood were obtained from the blood bank and analyzed for the presence of lymphocytes. Results: Prior transfusion did not affect karyotype results. A nonmosaic abnormal karyotype was found in 3 of the 10 patients. A fourth patient's karyotype was 45,X/47,XXX. This mosaicism was constitutive and consistent as demonstrated by a follow-up chromosome analysis. All other abnormal karyotypes were consistent with the dysmorphic phenotype. Randomly selected metaphases did not show any differences in the identifiable heteromorphic markers in all 10 patients. Although there was a $50 \%$ chance of patients receiving blood from a donor of opposite sex, there were no instances in which cells with a karyotype of the opposite sex were found in the patients' blood. The irradiated and filtered cultured donor blood samples did not show any metaphases. However, metaphases were seen in the cultures from nonfiltered and nonirradiated donor blood. Conclusions: Based on these results one does not need to delay karyotyping babies who have had blood transfusions. Packed red cell transfusion in newborns does not compromise the accuracy of chromosome analysis in our study even with multiple transfusions. Genetics in
\end{abstract}

Medicine, 2001:3(4):314-317.

Key Words: dysmorphic, cytogenetics, red blood cells, mosaicism, karyotype, transfusion

Critically ill neonates frequently require transfusions with a variety of blood products, and often receive multiple transfusions. Chromosome evaluation is a crucial part of the diagnostic evaluation of newborns with multiple congenital anomalies. Many of these critically ill infants receive blood transfusion before the need for chromosome analysis is determined. In many cases, the clinical management is dependent on results of chromosome analysis and it may become imperative to perform cytogenetic analysis on infants who have had transfusion. There is a long-standing tradition in pediatrics of not performing chromosome analysis after transfusion. This

\footnotetext{
From the ${ }^{1}$ Department of Pediatrics, ${ }^{2}$ Department of Pathology, Medical College of Georgia Augusta, Georgia; ${ }^{3}$ Department of Pediatrics, Metro Health Medical Center, Cleveland, Ohio.

Anita Kulharya, PhD, Department of Pediatrics, BG 1071B, Medical College of Georgia, Augusta, GA 30912.

Received: March 20, 2001

Accepted: April 9, 2001.
}

creates a frequent dilemma in clinical practice. This study was undertaken to determine whether any discrepancies are observed in the karyotype of newborns who had had transfusions with packed red cells.

\section{METHODS}

We performed a retrospective review of the medical records of all neonates who had chromosome analysis between June 1995 and June 1998. We identified 10 newborns who had chromosome analysis subsequent to blood transfusion. The age of these neonates ranged from 2 days to 3 weeks. The tests had been ordered because of dysmorphic features and/or multiple congenital anomalies. These 10 infants had been transfused two or more times. The time of transfusion ranged from 1 day to 10 days prior to being sampled for chromosome analysis. All the infants had received packed red cells. Seven of the ten patients had received blood products that had been irradiated and/or filtered. None of them had received whole blood. 
Routine cytogenetic analysis of phytohemagglutinin-stimulated 48-hour and/or 72-hour cultures had been performed on all 10 patients. Fluorescence in situ hybridization (FISH) had been performed on patients for $\operatorname{del}(22)$ syndrome and another patient that was referred for possible trisomy 13. In addition, from each of these 10 patients, 10 metaphases were randomly photographed. Heteromorphic markers were compared in these 10 metaphases for any discrepancy.

The irradiation and filtration of blood is done according to guidelines published by American Association of Blood Banks. ${ }^{1}$ For irradiation of blood products, the components are placed upside down in a stainless steel canister, which is placed in the sample chamber of Gammacell 1000 irradiator. The samples are irradiated for 4.5 minutes at $2500 \mathrm{rad}$. The filtration is generally carried out directly at the time of blood donation through a leukocyte depletion filter (PALL) attached to the collection unit. Samples of filtered/irradiated and nonfiltered blood were obtained from the blood bank and were cultured according to standard practice. Blood smear slides were also made from these donor samples. These slides were stained with Wright's stain and analyzed for the presence of lympho- cytes. Two slides were analyzed in each of these samples using a $20 \times$ objective.

\section{RESULTS}

A nonmosaic abnormal karyotype was found in 3 of the 10 patients. Two of these three patients received multiple transfusions with nonirradiated/nonfiltered packed red cells. A fourth patient's karyotype was 47,XXX[46]/45,X[4]. Follow-up chromosome analysis in this patient a year after the first cytogenetic analysis showed that this mosaicism was constitutive with a consistent degree of mosaicism. The results of our study are summarized in Table 1.

All abnormal karyotypes were consistent with the dysmorphic phenotype. The remaining six patients showed a normal karyotype with no evidence of any mosaicism. The karyotypic sex of each patient was consistent with the phenotypic sex, with no instances of low-level mosaicism for discordant karyotypic sex. Given the male to female donor ratio in our blood bank of approximately $1: 1$, the probability of any patient being transfused with blood from a donor of opposite sex is $50 \%$. The

Table 1

Results from cytogenetic analysis on 10 patients with packed red cell transfusions

\begin{tabular}{|c|c|c|c|}
\hline Indication for chromosome analysis & No. of transfusions & $\begin{array}{l}\text { Transfusion interval before } \\
\text { cytogenetic testing }\end{array}$ & Karyotype ( 20 cells) \\
\hline Tetralogy of Fallot and pulmonary atresia & $\begin{array}{c}2 \\
\text { (irradiated) }\end{array}$ & 1 day & $46, \mathrm{XX}$ \\
\hline Diaphragmatic hernia, polydactyly, and bifid thumb & $\begin{array}{c}2 \\
\text { (irradiated) }\end{array}$ & 10 days & $46, \mathrm{XY}$ \\
\hline Gonadal dysgenesis & 2 & 2 days & $\begin{array}{l}47, \mathrm{XXX}[46] / 45, \mathrm{X}[4] \\
(50 \text { cells })\end{array}$ \\
\hline MCA with cardiac and renal anomalies & $\begin{array}{c}1 \\
\text { (irradiated and filtered) }\end{array}$ & 3 days & $46, \mathrm{XX}$ \\
\hline Possible DiGeorge syndrome & $\begin{array}{c}2 \\
\text { (irradiated and filtered) }\end{array}$ & 1-2 days & $46, \mathrm{XX}$ \\
\hline Down syndrome & 10 & $1-10$ days & $47, \mathrm{XX},+21$ \\
\hline Possible DiGeorge syndrome & $\begin{array}{c}8 \\
\text { (irradiated and filtered) }\end{array}$ & 1 day & $\begin{array}{l}\text { 46,XY.ish del(22) } \\
(\mathrm{q} 11.2 \mathrm{q} 11.2) \\
(\mathrm{D} 22 \mathrm{~S} 75-)\end{array}$ \\
\hline
\end{tabular}

PDA, patent ductus arteriosus; MCA, multiple congenital anomalies. 
observation of no instances of even mosaic discordant karyotypic sex in any of our 10 patients is highly significant, with a likelihood of $<0.001$. Scrutiny of 10 randomly photographed metaphases did not demonstrate any differences in the identifiable heteromorphic markers, from cell to cell in all 10 patients.

No metaphases were seen in the irradiated and filtered cultured donor bloods. However, metaphases were seen in the cultures from nonfiltered and from nonirradiated donor blood. Microscopic analysis of the irradiated and/or filtered donor blood smears did not show any lymphocytes. Occasional mature lymphocytes were, however, seen in the smears from the donor blood sample that was neither filtered nor irradiated (Fig. 1).

\section{DISCUSSION}

It has been estimated that annually $80 \%$ of the 38,000 neonates with birth weight $<1500 \mathrm{~g}$ will receive multiple red blood cell transfusions. ${ }^{2}$ Many of these are newborns with congenital

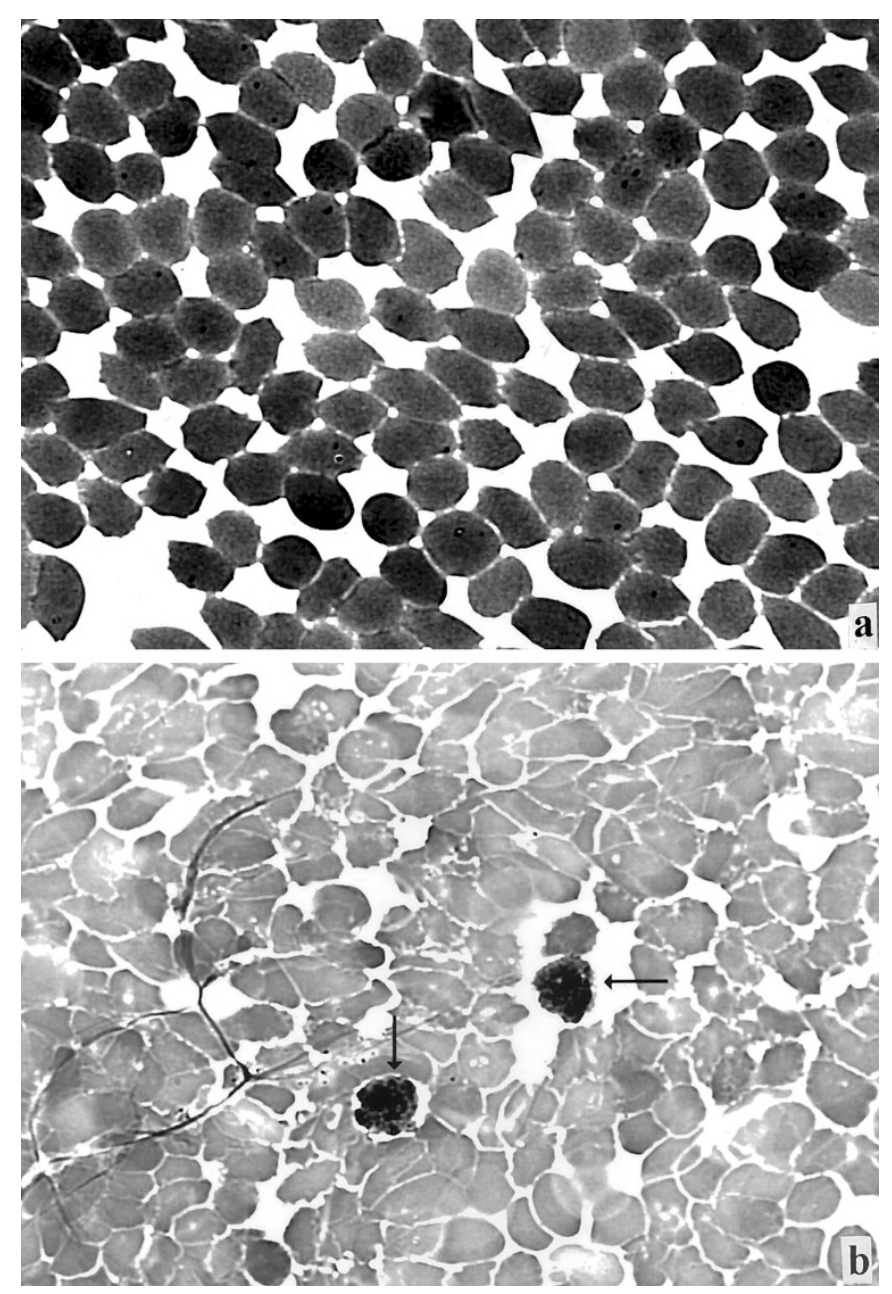

Fig. 1 (a) Smear of random donor blood that has been irradiated and filtered, demonstrating no white cells. (b) Smear from donor blood that has not been treated, demonstrating occasional mature lymphocytes (shown by arrows). anomalies and/or dysmorphic features. Sometimes the need for chromosome testing does not become apparent until after the neonate has had one or more transfusions, creating a dilemma. There is a long-standing tradition that advises against chromosome testing of babies who have received blood transfusion, but we were unable to find this recommendation in any of the standard pediatric or neonatal text books nor in a MEDLINE search.

The results of our study demonstrate that transfusion of sick neonates with nonirradiated or nonfiltered packed red cells even including untreated packed red cells, produces no adverse effect on the accuracy of karyotypes of these patients. Although this is an isolated study with a small sample size, the correlation of the clinical phenotype with the karyotype in three patients with abnormal results is unequivocal. One of these three patients had 10 transfusions with nonirradiated packed red blood cells and yet had a nonmosaic abnormal karyotype consistent with the clinical diagnosis. The FISH studies performed in some of our cases for various reasons also demonstrated unambiguous results consistent with the requesting diagnosis. The failure to find cells of the opposite sex in any of our patients studied is also evidence of lack of interference from transfusion.

Although the presence of mitogen responsive white cells was documented in smears as well as in cultured cells of nontreated donor cells, these leukocytes did not interfere with the accuracy of diagnosis in these three patients. The lack of any discrepancy in the heteromorphic markers from cell to cell in 10 randomly selected metaphases from each of these patients was strong evidence of lack of any mosaicism, even if they had been transfused with same-sex donor blood.

Proliferating lymphocytes have been shown to persist for a mean of 2.0 days in adult female patients transfused with packed red cells from male donors. Adams et al. ${ }^{3}$ used polymerase chain reaction to amplify a segment of repetitive sequences in Y chromosome to show that $>20,000$ total male cells were present in these female patient's circulation. In another study by Schechter et al., ${ }^{4}$ the authors found circulating donor lymphocytes up to a week in adult patients who had transfusion of moderate to large amounts of blood. Hutchinson et al. ${ }^{5}$ in a study compared the presence of donor cells in exchange transfusion with donor blood from blood bank versus fetal transfusion of maternal cells in newborns. They found that 10 of the 48 infants receiving donor blood transfusion demonstrated donor cells ranging in frequency from 1 to $9 \%$, 2-4 weeks posttransfusion. Three of these 10 infants showed persistence of donor cells 6-8 weeks posttransfusion with a frequency of $1-4 \%$. However, in the study the patients were transfused with whole blood that was not older than 24 hours. Also, the study was conducted 30 years ago. Since then, transfusion practices as well as donor blood treatments have undergone several changes. The likely explanation for the absence of any mosaicism in our patients may be a rapid elimination of the donor lymphocytes from the host. It is also likely that the stored lymphocytes have a poor response to mitogen. Another explanation may be that the survival of the lymphocyte de- 
creases with increased storage time, which is up to 35 days (with citrate phosphate dextrose adenine-1 red blood cell preservative) at our institution. Also, the persistent donor lymphocytes may be diluted to the extent that they are not detected in a routine analysis.

While the possibility of finding some type of mosaicism (normal/abnormal or sex chromosome discordance) remains when nontreated blood products are used for transfusion, the chances of karyotypic discrepancy are extremely small. If any mosaicism is detected on first cytogenetic analysis, the chromosome analysis must be repeated after $8-10$ weeks posttransfusion, or another tissue such as skin be analyzed cytogenetically. Recently, in neonatology, the trend has been toward transfusing with leukocyte-depleted blood, thus making the chromosome analysis even more risk free. Our study demonstrates that, although there is a theoretic possibility of some confusion of karyotypic results among patients who have been transfused, there is no practical prohibition against doing so.

\section{References}

1. Widmann FK, editor. Standards for blood banks and transfusion services. 14th ed. Arlington, VA: American Association of Blood Banks, 1991.

2. Ringer SA, Richardson DK, Sacher RA, Keszler M, Churchill WH. Variations in transfusion practice in neonatal intensive care. Pediatrics 1998;101:194-200.

3. Adams PT, Davenport RD, Reardon DA, Roth MS. Detection of circulating donor white blood cells in patients receiving multiple transfusions. Blood 1992;80:551-555.

4. Schechter GP, Whang-Peng J, McFarland W. Circulation of donor lymphocytes after blood transfusion in man. Blood 1977;49:651-656.

5. Hutchinson DL, Turner JH, Schlesinger ER. Persistence of donor cells in neonates after fetal and exchange transfusion. Am J Obstet Gynecol 1971;109:281-284. 